PROCEEDINGS OF THE

AMERICAN MATHEMATICAL SOCIETY

Volume 127, Number 6, Pages 1571-1574

S 0002-9939(99)05231-4

Article electronically published on February 17, 1999

\title{
THE WEIERSTRASS APPROXIMATION THEOREM AND A CHARACTERIZATION OF THE UNIT CIRCLE
}

\author{
J. BOCHNAK AND W. KUCHARZ
}

(Communicated by Ron Donagi)

\begin{abstract}
We study real algebraic morphisms from nonsingular real algebraic varieties $X$ with $\operatorname{dim} X \geq 1$ into nonsingular real algebraic curves $C$. We show, among other things, that the set of real algebraic morphisms from $X$ into $C$ is never dense in the space of all $\mathcal{C}^{\infty}$ maps from $X$ into $C$, unless $C$ is biregularly isomorphic to a Zariski open subset of the unit circle.
\end{abstract}

An affine real algebraic variety is a locally ringed space isomorphic to an algebraic subset of $\mathbb{R}^{n}$ (for some $n$ ) endowed with the Zariski topology and the sheaf of $\mathbb{R}$ valued regular functions. For basic information on real algebraic varieties the reader may refer to [2]. Recall that every quasiprojective real algebraic variety is in fact affine [2, Proposition 3.2.10, Theorem 3.4.4]. Given two affine real algebraic varieties $X$ and $Y$, we denote by $\mathcal{R}(X, Y)$ the set of all regular maps (that is, morphisms of locally ringed spaces) from $X$ into $Y$. We assume that $X$ and $Y$ are nonsingular and regard $\mathcal{R}(X, Y)$ as a subset of the space $\mathcal{C}^{\infty}(X, Y)$ of all $\mathcal{C}^{\infty}$ maps from $X$ into $Y$ endowed with the $\mathcal{C}^{\infty}$ compact-open topology (the weak $\mathcal{C}^{\infty}$ topology in the terminology used in [7]). It is natural to study the size of $\mathcal{R}(X, Y)$ in $\mathcal{C}^{\infty}(X, Y)$ and several papers have already been devoted to this problem [2], [3], [4], [5]. In the present note we prove the following result, which can be viewed as a version, in a new setting, of the classical Weierstrass approximation theorem.

Theorem 1. Given an affine nonsingular irreducible real algebraic curve $C$, the following conditions are equivalent:

(a) The curve $C$ is biregularly isomorphic to a Zariski open subset of the unit circle $S^{1}=\left\{(x, y) \in \mathbb{R}^{2} \mid x^{2}+y^{2}=1\right\}$

(b) The set $\mathcal{R}(V, C)$ is dense in $\mathcal{C}^{\infty}(V, C)$ for every compact affine nonsingular real algebraic curve $V$;

(c) There exists an affine nonsingular real algebraic variety $X$ such that $\operatorname{dim} X \geq$ 1 and $\mathcal{R}(X, C)$ is dense in $\mathcal{C}^{\infty}(X, C)$.

To prevent possible confusion let us say explicitly that throughout this note the adjective compact always refers to the Euclidean topology on the real algebraic varieties.

Received by the editors July 29, 1996.

1991 Mathematics Subject Classification. Primary 14G30, 14C99.

Both authors were partially supported by NATO Collaborative Research Grants Programme CRG 960011.

The second author was partially supported by NSF Grant DMS-9503138.

(C)1999 American Mathematical Society 
Before giving a proof of Theorem 1 we make a few remarks. For every affine nonsingular irreducible real algebraic curve $V$ there exists a unique (up to biregular isomorphism over $\mathbb{R}$ ) projective nonsingular irreducible complex algebraic curve $V_{\mathbb{C}}$ defined over $\mathbb{R}$ such that $V$ is biregularly isomorphic to a Zariski open subset of the set of real points $V_{\mathbb{C}}(\mathbb{R})$ of $V_{\mathbb{C}}$ (here $V_{\mathbb{C}}(\mathbb{R})$ is regarded as a projective real algebraic variety). We denote by $g(V)$ the genus of $V_{\mathbb{C}}$. Every regular map $f: V \longrightarrow W$ of affine nonsingular irreducible real algebraic curves extends in a unique way to a (complex) regular map $f_{\mathbb{C}}: V_{\mathbb{C}} \longrightarrow W_{\mathbb{C}}$. Let us denote by $\mathcal{R}^{*}(V, W)$ the set of all nonconstant regular maps from $V$ into $W$. By applying the remarks above and some classical theorems on projective complex algebraic curves we obtain information on the set $\mathcal{R}^{*}(V, W)$. Namely:

(i) By the theorem of de Franchis [8, p. 227], if $g(W) \geq 2$, then the set $\mathcal{R}^{*}(V, W)$ is finite,

(ii) The Hurwitz-Riemann formula [6, p. 140] implies that if $g(W)=1$, then each map in $\mathcal{R}^{*}(V, W)$ has at most $2 g(V)-2$ critical points.

Proof of Theorem 1. Assume that (a) holds. It is known that the set $\mathcal{R}\left(V, S^{1}\right)$ is dense in $\mathcal{C}^{\infty}\left(V, S^{1}\right)$ for every compact affine nonsingular real algebraic curve $V$ (cf. [4, Proposition 6.1, Theorem 1.6], where the problem of determining the size of $\mathcal{R}\left(X, S^{1}\right)$ in $\mathcal{C}^{\infty}\left(X, S^{1}\right)$ is completely solved for an arbitrary compact affine nonsingular real algebraic variety $X$ ). If $C$ is biregularly isomorphic to a Zariski open subset of $S^{1}$, different from $S^{1}$, then $C$ is biregulary isomorphic to a Zariski open subset of $\mathbb{R}$ and hence the set $\mathcal{R}(X, C)$ is dense in $\mathcal{C}^{\infty}(V, C)$ for every compact affine nonsingular real algebraic variety $X$. Thus (b) is proved.

It is obvious that (b) implies (c). We now prove that (c) implies (a). Suppose that the set $\mathcal{R}(X, C)$ is dense in $\mathcal{C}^{\infty}(X, C)$ for some affine nonsingular real algebraic variety $X$ with $\operatorname{dim} X \geq 1$. We shall show $g(C)=0$, which is equivalent to (a). To this end let us choose a nonsingular irreducible real algebraic curve $Z$ in $X$.

We have $g(C) \leq 1$ since otherwise the set $\mathcal{R}^{*}(Z, C)$ would be finite (cf. (i)) and therefore no map $f$ in $\mathcal{C}^{\infty}(X, C)$ whose restriction $f \mid Z$ is not in $\mathcal{R}(Z, C)$ could be approximated by regular maps from $X$ into $C$.

Hence it remains to exclude the case $g(C)=1$. Choose a $\mathcal{C}^{\infty}$ map $h: X \longrightarrow C$ such that $h \mid Z$ has at least $2 g(Z)-1$ critical points, each of multiplicity exactly 2 (in particular, $h \mid Z$ has in local coordinates either a local maximum or a local minimum at each critical point). If $g(C)=1$, then it follows from (ii) that $h \mid Z$ (and hence $h$ ) cannot be approximated by regular maps with values in $C$. Thus $g(C)=0$ is proved.

The following characterization of the unit circle is an immediate consequence of Theorem 1.

Corollary 2. Let $C$ be a compact affine nonsingular irreducible real algebraic curve. Then the following conditions are equivalent:

(a) The curve $C$ is biregularly isomorphic to $S^{1}$;

(b) The set $\mathcal{R}(V, C)$ is dense in $\mathcal{C}^{\infty}(V, C)$ for every compact affine nonsingular real algebraic curve $V$;

(c) There exists an affine nonsingular real algebraic variety $X$ such that $\operatorname{dim} X \geq$ 1 and $\mathcal{R}(X, C)$ is dense in $\mathcal{C}^{\infty}(X, C)$. 
It would be interesting, but probably hard, to find a generalization of Theorem 1 and Corollary 2 with $C$ replaced by a higher dimensional real algebraic variety. In this direction we only have the following result.

Theorem 3. Let $M$ be a closed connected $\mathcal{C}^{\infty}$ manifold with $\operatorname{dim} M \geq 1$. Then there exists an affine nonsingular real algebraic variety $Y$ such that $M$ is diffeomorphic to $Y$ and the set $\mathcal{R}(X, Y)$ is never dense in $\mathcal{C}^{\infty}(X, Y)$, where $X$ is an arbitrary affine nonsingular real algebraic variety with $\operatorname{dim} X \geq 1$.

We shall need the following observation.

Lemma 4. Let $Y$ be an affine nonsingular irreducible real algebraic variety. Assume that there exists a nonconstant regular map $\varphi: Y \longrightarrow C$ into an affine nonsingular irreducible real algebraic curve $C$ with $g(C) \geq 2$. Then the set $\mathcal{R}(X, Y)$ is never dense in $\mathcal{C}^{\infty}(X, Y)$, where $X$ is an arbitrary affine nonsingular real algebraic variety with $\operatorname{dim} X \geq 1$.

Proof. Since $Y$ is irreducible and $\varphi: Y \longrightarrow C$ is nonconstant, we can choose a sequence $\left\{U_{n}\right\}$ of nonempty open (in the Euclidean topology) subsets of $Y$ such that the subsets $\varphi\left(U_{n}\right)$ of $C$ are pairwise disjoint.

Suppose now that $\mathcal{R}(X, Y)$ is dense in $\mathcal{C}^{\infty}(X, Y)$ for some affine nonsingular real algebraic set $X$ with $\operatorname{dim} X \geq 1$. Let $Z$ be a nonsingular irreducible algebraic curve in $X$ and let $z$ be a point in $Z$. Since $\mathcal{R}(X, Y)$ is dense in $\mathcal{C}^{\infty}(X, Y)$, we can find a sequence $\left\{\varphi_{n}\right\}$ of regular maps $\varphi_{n}: Z \longrightarrow Y$ such that $\varphi_{n}(z)$ is in $U_{n}$ and the composition $\varphi \circ \varphi_{n}$ is nonconstant. Then $\left\{\varphi \circ \varphi_{n}\right\}$ is an infinite family of distinct elements of $\mathcal{R}^{*}(Z, C)$, which is impossible in view of (i). This contradiction completes the proof.

Proof of Theorem 3. Let $C$ be a compact connected affine nonsingular real algebraic curve with $g(C) \geq 2$. Let $f: M \longrightarrow C$ be a nonconstant $\mathcal{C}^{\infty}$ map. Then given a neighborhood $\mathcal{U}$ of $f$ in $\mathcal{C}^{\infty}(M, C)$, one can find an affine nonsingular real algebraic variety $Y$, a $\mathcal{C}^{\infty}$ diffeomorphism $\sigma: M \longrightarrow Y$, and a regular map $\varphi: Y \longrightarrow C$ such that $\varphi \circ \sigma$ is in $\mathcal{U}$ (one embeds $M$ in $\mathbb{R}^{n}$, for some $n$, and applies [1, Theorem 2.8.4], observing first that the bordism class of $(f, M)$ in the unoriented bordism group of $C$ is algebraic [1, Lemma 2.7.1]). We may assume that $\varphi$ is nonconstant by taking $\mathcal{U}$ sufficiently small. Moreover, $Y$ must be irreducible since $M$ is connected. We now complete the proof of Theorem 3 by applying Lemma 4.

We say that a closed $\mathcal{C}^{\infty}$ manifold $M$ admits a Weierstrass algebraic model if there exists an affine nonsingular real algebraic variety $Y$ such that $M$ is diffeomorphic to $Y$ and the set $\mathcal{R}(X, Y)$ is dense in $\mathcal{C}^{\infty}(X, Y)$ for some affine nonsingular real algebraic variety $X$ with $\operatorname{dim} X \geq 1$. It is likely that only very few closed $\mathcal{C}^{\infty}$ manifolds admit Weierstrass algebraic models. We conjecture that among closed connected orientable $\mathcal{C}^{\infty}$ surfaces only the 2 -sphere $S^{2}$ and the torus $S^{1} \times S^{1}$ admit Weierstrass algebraic models.

\section{REFERENCES}

[1] S. Akbulut and H. King, Topology of Real Algebraic Sets, Math. Sci. Research Institute publ. 25, Springer, New York, Berlin, Heidelberg, 1992. MR 94m:57001

[2] J. Bochnak, M. Coste and M.-F. Roy, Géométrie Algébrique Réelle, Springer, Berlin and New York, 1987. MR 90b:14030 
[3] J. Bochnak and W. Kucharz, On real algebraic morphisms into even-dimensional spheres, Ann. of Math. 128 (1988), 415-433. MR 89k:57060

[4] J. Bochnak and W. Kucharz, Algebraic models of smooth manifolds, Invent. Math. 97 (1989), 585-611. MR 91b:14076

[5] J. Bochnak and W. Kucharz, Elliptic curves and real algebraic morphisms, J. Algebraic Geometry 2 (1993), 635-666. MR 94e:14072

[6] O. Forster, Lectures on Riemann Surfaces, Springer, Berlin, Heidelberg, New York, 1981. MR 83d:30046

[7] M. W. Hirsch, Differential Topology, Springer, Berlin, Heidelberg, New York, 1976. MR 56:6669; MR 96c:57001

[8] B. Mazur, Arithmetic on curves, Bull. Amer. Math. Soc. 14 (1986), 209-260. MR 88e:11050

Department of Mathematics, Vrije Universiteit, De Boelelaan 1081, 1081 HV AmsTERDAM, The Netherlands

E-mail address: bochnak@cs.vu.nl

Department of Mathematics, University of New Mexico, Albuquerque, New Mexico 87131

E-mail address: kucharz@math.unm.edu 